Pacific Journal of Mathematics

A CONVERGENT GRADIENT PROCEDURE IN PREHILBERT 


\title{
A CONVERGENT GRADIENT PROCEDURE IN PREHILBERT SPACES
}

\author{
E. K. BLUM
}

In this paper, we present a new method of approximating the minimum of a functional, $J$, defined on a prehilbert space and subject to constraints of the form $\phi_{i}(x)=0,1 \leqq i \leqq p$, where the $\phi_{i}$ are also functionals on the space. The method generates a convergent sequence of approximations using the gradients of $J$ and $\phi_{i}$. However, it is not a steepest descent procedure with respect to $J$. A theorem is proven which establishes the convergence of the approximating sequence to the minimum.

The literature on extremal problems in abstract spaces is fairly extensive. We refer to the bibliographies in [1], [5] for a partial list. That part of the literature which deals with approximation procedures for finding extrema subject to various constraints is also extensive. Again, see [1], [5] and also [3] for bibliographies. In particular, gradient-type methods have received considerable attention recently in a variety of contexts as in [2], [7], [6] to mention a few. In this paper, we present a new method of approximating the minimum of a functional, $J$, defined on a prehilbert space and subject to equality constraints. The method generates a convergent sequence of approximations using gradients but it is not of the steepest descent type with respect to $J$.

2. Preliminary remarks, Let $H$ be a prehilbert space. For $u, v, \in H$, we denote their inner product by $\langle u, v\rangle$ and $\|u\|^{2}=\langle u, u\rangle$.

Let $J$ and $\psi_{i}, 1 \leqq i \leqq p$, be real functionals defined on some subset in $H$. We shall say that $u^{*} \in H$ is a solution of the minimization problem defined by $\left\{J, \psi_{i}\right\}$ if $\psi_{i}\left(u^{*}\right)=0,1 \leqq i \leqq p$ and $J\left(u^{*}\right) \leqq$ $J(u)$ for those $u$ in a neighborhood of $u^{*}$ which satisfy the constraints $\psi_{i}(u)=0,1 \leqq i \leqq p$.

By the gradient of $J$ at $u$ we mean an element of $H$, designated by $\nabla J(u)$, such that for all $\Delta u$ in some neighborhood of $0 \in H$,

$$
J(u+\Delta u)-J(u)=\langle\nabla J(u), \Delta u\rangle+\varepsilon(u, \Delta u),
$$

where $|\varepsilon(u, \Delta u)| /\|\Delta u\| \rightarrow 0$ as $\Delta u \rightarrow 0$. Similarly, $\nabla \psi_{i}(u)$ denotes the gradient of $\psi_{i}$ at $u$.

If $f$ is a real functional defined in a neighborhood of $u \in H$ and if $d f(u ; h)=\lim _{s \rightarrow 0}(f(u+s h)-f(u)) / s$ exists for all $h \in H$, we call $d f(u ; h)$ the weak differential of $f$ at $u$ with respect to $h$. The relation 
between $d f(u ; h)$ and $\langle\nabla f(u), h\rangle$ is well-known [8].

Now suppose that $\nabla J(u)$ and $\nabla \psi_{i}(u), 1 \leqq i \leqq p$, exist at $u$. Suppose further that the $p \times p$ Gram matrix,

$$
D(u)=\left(\left\langle\nabla \psi_{i}(u), \nabla \psi_{j}(u)\right\rangle\right),
$$

is nonsingular. Let $\gamma$ be the $p$-dimensional row vector

$$
\left(\left\langle\nabla J(u), \nabla \psi_{1}(u)\right\rangle, \cdots,\left\langle\nabla J(u), \nabla \psi_{p}(u)\right\rangle\right)
$$

and define $\lambda=\left(\lambda_{1}, \cdots, \lambda_{p}\right)$ as the vector $\lambda=\gamma D^{-1}$. Then, as is wellknown, the "projection" of $\nabla J$ on the subspace $G \subset H$ spanned by $\left\{\nabla \psi_{i}(u) \mid 1 \leqq i \leqq p\right\}$ is given by $\nabla J_{G}(u)=\sum_{1}^{p} \lambda_{j} \nabla \psi_{j}(u)$. The component of $\nabla J$ orthogonal to $G$ is

$$
\nabla J_{T}(u)=\nabla J(u)-\nabla J_{G}(u) .
$$

If $u^{*}$ is a solution of the minimization problem defined by $\left\{J, \psi_{i}\right\}$ and $\nabla J(u), \nabla \psi_{i}(u)$ exist as continuous functions of $u$ in a neighborhood of $u^{*}$ with $D\left(u^{*}\right)$ nonsingular, then by the Lagrange multiplier rule [4], [5], [8] it follows that $\nabla J_{T}\left(u^{*}\right)=0$.

In the next section, we shall use this necessary condition as the basis for a gradient method of obtaining successive approximations which converge to $u^{*}$ when certain regularity conditions hold in a neighborhood of $u^{*}$. With obvious modifications, the method can also be applied to the simpler problem of finding a solution of the system $\left\{\psi_{i}(u)=0\right\}$. In this context, it generalizes a method given in [7] for finite-dimensional spaces.

3. The gradient method. For any $u \in H$, we shall use the notation " $\bar{u}$ " to denote the normalized vector $(1 /\|u\|) u$.

DEFINITION. Let $u^{*}$ be a solution of the minimization problem defined by $\left\{J, \psi_{i}\right\} . u^{*}$ is a regular minimum if there is a neighborhood, $N=N\left(u^{*}\right)$, of $u^{*}$ in which the following conditions are satisfied:

(1) $\nabla J(u)$ exists as a continuous function of $u$ and $\nabla J_{\theta}(u) \neq 0$ for $u \in N$

(2) $\nabla \psi_{i}(u), 1 \leqq i \leqq p$, exists as a continuous function of $u$ and $\nabla \psi_{i}(u) \neq 0$ for $u \in N$;

(3) For each $u \in N$, the matrix $D(u)$ is nonsingular;

(4) For $\theta(u)=\arcsin \left(\left\|\nabla J_{\tau}(u)\right\| /\|\nabla J(u)\|\right)$, the gradient $\nabla \theta(u)$ exists and $\|\nabla \theta(u)\|>a>0, u \neq u^{*}$. At $u^{*}$ the weak differential $d \theta\left(u^{*} ; h\right)$ exists and $\langle\nabla \theta(u), h\rangle \rightarrow d \theta\left(u^{*}, h\right)$ as $u \rightarrow u^{*}$. 
(5) For $u=\left(u^{*}+\Delta u\right) \in N, \Delta u \neq 0$, let

$$
\begin{aligned}
\alpha_{i}=\alpha_{i}(u) & =\arccos \left\langle\overline{\nabla \psi_{i}(u)}, \overline{\Delta u}\right\rangle, 1 \leqq i \leqq p, \\
\alpha_{0}=\alpha_{0}(u) & =\arccos \left\langle\overline{\nabla J_{T}(u)}, \overline{\Delta u}\right\rangle, \\
\beta & =\beta(u)=\arccos \langle\overline{\nabla \theta(u)}, \overline{\Delta u}\rangle, \\
\gamma=\gamma(u) & =\arccos \left\langle\overline{\nabla \theta(u)}, \overline{\nabla J_{T}(u)}\right\rangle .
\end{aligned}
$$

There exist constants $a_{1}>0$ and $a_{2}>0$ such that

$$
\sum_{1}^{p} \cos ^{2} \alpha_{i}+\cos \alpha_{0} \cos \beta /|\cos \gamma|>a_{1}
$$

and $|\cos \gamma|>a_{2}$ for all $u \in N$ except possibly $u^{*}$. A neighborhood such as $N$ is called a regular neighborhood of $u^{*}$.

REMARK. $\theta\left(u^{*}\right)=0$ by the necessary condition for a minimum. If $u^{*}$ is the unique minimum, this condition is also sufficient under the assumptions required in the multiplier rule.

LEMma. Let $u^{*}$ be the unique solution of the minimization problem defined by $\left\{J, \psi_{i}\right\}$ and let $N$ be a regular neighborhood of $u^{*}$. For $u=\left(u^{*}+\Delta u\right) \in N, \Delta u \neq 0$, let

$$
\begin{aligned}
& h_{G}=h_{\theta}(u)=-\sum_{i=1}^{p}\left(\psi_{i}(u) /\left\|\nabla \psi_{i}(u)\right\|\right) \overline{\nabla \psi_{i}(u)}, \\
& h_{T}=h_{T}(u)=-\left(1 /\left\|\nabla J_{G}(u)\right\| \cdot\left|\left\langle\nabla \theta(u), \overline{\nabla J_{T}(u)}\right\rangle\right|\right) \nabla J_{T}(u),
\end{aligned}
$$

and $h=h_{G}+h_{T}$. Then there exists positive constants $k$, $d$ and $r$, with $k<1$, such that for all $u$ with $0<\|\Delta u\|<r$, $u$ is in $N$ and $\left\|u+s h-u^{*}\right\|<k\|\Delta u\|$ whenever $d / 2<s<d$.

Proof. By the definition of gradient,

$$
\psi_{i}(u)=\psi_{i}(u)-\psi_{i}\left(u^{*}\right)=\left\langle\nabla \psi_{i}\left(u^{*}\right), \Delta u\right\rangle+\varepsilon_{i}, 1 \leqq i \leqq p,
$$

where $\varepsilon_{i}=\varepsilon_{i}\left(u^{*}, \Delta u\right)$ is such that $\left\|\varepsilon_{i}\right\| /\|\Delta u\| \rightarrow 0$ as $\Delta u \rightarrow 0$.

(For convenience, we shall adopt the notational convention that throughout the proof any quantity designated by $\varepsilon$ with appropriate subscripts or superscripts is such that $\|\varepsilon\| /\|\Delta u\| \rightarrow 0$ as $\Delta u \rightarrow 0$. We shall make no further mention of this property.)

Now, by the continuity of $\nabla \psi_{i}$ at $u^{*}$ (condition 2 of the definition), $\psi_{i}(u)=\left\langle\nabla \psi_{i}(u), \Delta u\right\rangle+\tilde{\varepsilon}_{i}$. Using these relations in (6), we obtain

$$
h_{G}=-\sum_{1}^{p}\left\langle\overline{\nabla \psi_{i}}(u), \Delta u\right\rangle \overline{V \psi_{i}(u)}+\varepsilon_{G} .
$$

Since $u+s h-u^{*}=\Delta u+s h_{G}+s h_{T}$, we have 
(9) $\left\|u+s h-u^{*}\right\|^{2}$

$$
=\|\Delta u\|^{2}+2 s\left\langle\Delta u, h_{G}\right\rangle+2 s\left\langle\Delta u, h_{T}\right\rangle+s^{2}\left(\left\|h_{G}\right\|^{2}+\left\|h_{T}\right\|^{2}\right) .
$$

From (8) it follows that

$$
\left\langle h_{\theta}, \Delta u\right\rangle=-\|\Delta u\|^{2} \sum_{1}^{p} \cos ^{2} \alpha_{i}+\left\langle\varepsilon_{G}, \Delta u\right\rangle,
$$

where the $\alpha_{i}$ are given in condition 5 of the definition. Applying the Schwarz inequality to (8) yields

$$
\left\|h_{G}\right\|^{2} \leqq\|\Delta u\|^{2}\left(p \sum_{1}^{p} \cos ^{2} \alpha_{i}+w_{G}(\Delta u)\right) .
$$

where $w_{\theta}(\Delta u) \rightarrow 0$ as $\Delta u \rightarrow 0$.

Using (7), we find

$$
\left\langle h_{r}, \Delta u\right\rangle=-\frac{\left\|\nabla J_{T}(u)\right\|}{\left\|\nabla J_{G}(u)\right\|} \frac{\|\Delta u\| \cos \alpha_{0}}{\|\nabla \theta(u)\||\cos \gamma|}=-\tan \theta(u) \frac{\|\Delta u\| \cos \alpha_{0}}{\|\nabla \theta(u)\||\cos \gamma|},
$$

where $\alpha_{0}$ and $\gamma$ are given in condition 5. By the properties of $\theta(u)$ in condition 4 and the definition of the weak differential, we have

$$
\tan \theta(u)=\tan \theta\left(u^{*}\right)+\sec ^{2} \theta\left(u^{*}\right)\langle\nabla \theta(u), \Delta u\rangle+\hat{\varepsilon}=\langle\nabla \theta(u), \Delta u\rangle+\hat{\varepsilon} .
$$

Since $\langle\nabla \theta(u), \Delta u\rangle=\|\nabla \theta(u)\|\|\Delta u\| \cos \beta$, we obtain

$$
\left\langle h_{T^{\prime}}, \Delta u\right\rangle=-\|\Delta u\|^{2}\left(\cos \alpha_{0} \cos \beta /|\cos \gamma|+w(\Delta u)\right),
$$

where $w(\Delta u) \rightarrow 0$ as $\Delta u \rightarrow 0$. Similarly, from (7) we see that

$$
\left\|h_{T}\right\|=\frac{\left\|\nabla J_{T}(u)\right\|}{\left\|\nabla J_{G}(u)\right\|} \frac{1}{\|\nabla \theta(u)\||\cos \gamma|}=\|\Delta u\|\left(\cos \beta / \cos \alpha+w_{T^{\prime}}\right)
$$

where $w_{T} \rightarrow 0$ as $\Delta u \rightarrow 0$.

Combining (9)-(13), we obtain

$$
\left\|u+s h-u^{*}\right\|^{2} \leqq\|\Delta u\|^{2}\left(\Phi_{1}(s)+2 s w_{1}(\Delta u)+s^{2} w_{2}(\Delta u)\right),
$$

where $w_{1}(\Delta u) \rightarrow 0$ and $w_{2}(\Delta u) \rightarrow 0$ as $\Delta u \rightarrow 0$ and

$$
\begin{aligned}
\Phi_{1}(s) & =1-2 b s+c_{1} s^{2}, \\
b & =\sum_{1}^{p} \cos ^{2} \alpha_{i}+\cos \alpha_{0} \cos \beta /|\cos \gamma|, \\
c_{1} & =p \sum_{1}^{p} \cos ^{2} \alpha_{i}+(\cos \beta / \cos \gamma)^{2} .
\end{aligned}
$$

If we replace $\Phi_{1}(s)$ by $\Phi(s)=1-2 b s+c s^{2}$, where

$$
c=c_{1}+\left(2 \sum_{1}^{p} \cos ^{2} \alpha_{i}+1+1 /|\cos \gamma|\right) /|\cos \gamma|,
$$

the inequality in (14) remains valid. Now, $0<\Phi(s)<1$ for 
$0<s<2 b / c$. Since $c \leqq p^{2}+(2 p+3) /|\cos \gamma|^{2}$ and, by condition 5 of the definition, $|\cos \gamma|>a_{2}>0$ and $b>a_{1}>0$, it follows that $0<\Phi(s)<1$ for $0<s<d$, where

$$
d=2 a_{1} a_{2}^{2} /\left(a_{2}^{2} p^{2}+2 p+3\right) .
$$

For $d / 2<s<d$ and $0<\|\Delta u\|<r$ with $r$ sufficiently small, there exists a constant $k^{2}$ such that $0<\Phi(s)+2 s w_{1}(\Delta u)+s^{2} w_{2}(\Delta u)<k^{2}<1$. Hence, $\left\|u+s h-u^{*}\right\|<k\|\Delta u\|$ whenever $d / 2<s<d$, which completes the proof.

The lemma serves as the basis of a convergent procedure for approximating $u^{*}$, as described in the following theorem.

THEOREm. Let $u^{*}, k, d$ and $r$ be as in the lemma. Let $u_{0} \in H$ be such that $\left\|u_{0}-u^{*}\right\|<r$. For every integer $n \geqq 0$ define

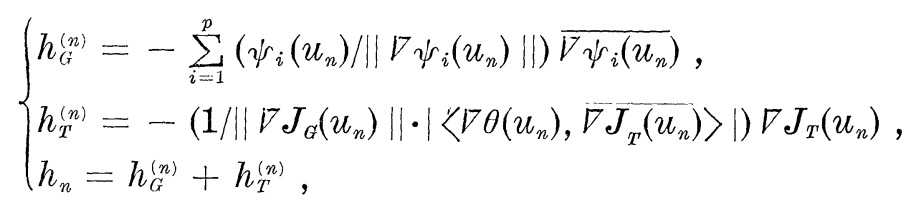

if $\nabla J_{T}\left(u_{n}\right) \neq 0$. If $\nabla J_{T}\left(u_{n}\right)=0$, take $h_{T}^{(n)}=0$. Further, define for $n \geqq 0$,

$$
u_{n+1}=u_{n}+s_{n} h_{n} \text {, where } d / 2<s_{n}<d .
$$

Then $\lim _{n \leftarrow \infty} u_{n}=u^{*}$ and $i n$ fact,

$$
\left\|u_{n}-u^{*}\right\|<k^{n}\left\|u_{0}-u^{*}\right\| \text {. }
$$

Proof. Since $\left\|u_{0}-u *\right\|<r, u_{0}$ satisfies the conditions on the point $u$ of the lemma. Comparing $h_{0}$ with $h$ and $s_{0}$ with $s$ of the lemma, we see immediately that $\left\|u_{1}-u^{*}\right\|<k\left\|u_{0}-u^{*}\right\|$. Since $k<1,\left\|u_{1}-u^{*}\right\|<r$ and the lemma can be applied at $u_{1}$ to obtain $\left\|u_{2}-u^{*}\right\|<k\left\|u_{1}-u^{*}\right\|<k^{2}\left\|u_{0}-u^{*}\right\|$. By induction, (18) is immediate and this establishes the theorem.

The application of this theorem to numerical procedures for solving minimization problems with equality constraints will be the subject of a forthcoming paper.

\section{REFERENCES}

1. H. Antosiewicz and W. Rheinboldt, Numerical analysis and functional analysis, Survey of Numerical Analysis, McGraw-Hill, 1962.

2. A. V. Balakrishnan, An operator theoretic formulation of a class of control problems and a steepest descent method of solution, J.S.I.A.M. Control, Ser. A, Vol. 1, No. 2, 1963. 
3. A. V. Balakrishnan and L. W. Neustadt, ed., Computing Methods in Optimization Problems, Academic Press, 1964.

4. E. K. Blum, Minimization of functionals with equality constraints, Abstract 64T381, Notices A. M. S., August 1964.

5. - Minimization of functionals with equality constraints, J. S. I. A. M. Control. Ser. A, vol. 3, No. 2, 1965, pp. 299-316.

6. A. Goldstein, Convex programming in Hilbert space, Bull. Amer. Math. Soc. 70, No. 5, September 1964, 709-710.

7. W. Hart and T. S. Motzkin, A composite Newton-Raphson gradient method for the solution of systems of equations, Pacific J. Math. 6 (1956), 691-707.

8. L. Liusternik and V. Sobolev, Elements of Functional Analysis, Ungar, 1961.

Received February 26, 1965.

WESLEYAN UNIVERSITY

Middletown, Connecticut 


\section{PACIFIC JOURNAL OF MATHEMATICS}

H. SAMELSON

Stanford University Stanford, California

R. M. BLUMENTHAL

University of Washington

Seattle, Washington 98105

\section{EDITORS}

\author{
*J. DugundJI \\ University of Southern California \\ Los Angeles, California 90007 \\ RICHARD ARENS \\ University of California \\ Los Angeles, California 90024
}

\section{ASSOCIATE EDITORS}

E. F. BeCKENBACH

B. H. NEUMANN

F. WOLF

K. YosidA

\section{SUPPORTING INSTITUTIONS}

UNIVERSITY OF BRITISH COLUMBIA

CALIFORNIA INSTITUTE OF TECHNOLOGY

UNIVERSITY OF CALIFORNIA

MONTANA STATE UNIVERSITY

UNIVERSITY OF NEVADA

NEW MEXICO STATE UNIVERSITY

OREGON STATE UNIVERSITY

UNIVERSITY OF OREGON

OSAKA UNIVERSITY

UNIVERSITY OF SOUTHERN CALIFORNIA
STANFORD UNIVERSITY

UNIVERSITY OF TOKYO

UNIVERSITY OF UTAH

WASHINGTON STATE UNIVERSITY

UNIVERSITY OF WASHINGTON

AMERICAN MATHEMATICAL SOCIETY CHEVRON RESEARCH CORPORATION TRW SYSTEMS

NAVAL ORDNANCE TEST STATION 


\section{Pacific Journal of Mathematics}

Vol. 18, No. 1

March, 1966

Edward Joseph Barbeau, Semi-algebras that are lower semi-lattices ...... 1

Steven Fredrick Bauman, The Klein group as an automorphism group

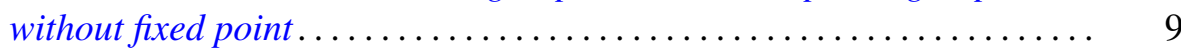

Homer Franklin Bechtell, Jr., Frattini subgroups and $\Phi$-central groups .... 15

Edward Kenneth Blum, A convergent gradient procedure in prehilbert

spaces ............................................

Edward Martin Bolger, The sum of two independent exponential-type random variables ...................................

David Wilson Bressler and A. P. Morse, Images of measurable sets .......

Dennison Robert Brown and J. G. LaTorre, A characterization of uniquely

divisible commutative semigroups........................

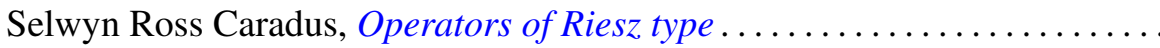

Jeffrey Davis and Isidore Isaac Hirschman, Jr., Toeplitz forms and ultraspherical polynomials ............................

Lorraine L. Foster, On the characteristic roots of the product of certain rational integral matrices of order two ......................

Alfred Gray and S. M. Shah, Asymptotic values of a holomorphic function

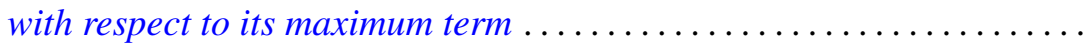

Sidney (Denny) L. Gulick, Commutativity and ideals in the biduals of

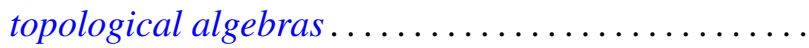

G. J. Kurowski, Further results in the theory of monodiffric functions

Lawrence S. Levy, Commutative rings whose homomorphic images are self-injective .

Calvin T. Long, On real numbers having normality of order $k$....

Bertram Mond, An inequality for operators in a Hilbert space. ...

John William Neuberger, The lack of self-adjointness in three-point boundary value problems ........................

C. A. Persinger, Subsets of $n$-books in $E^{3}$

Oscar S. Rothaus and John Griggs Thompson, A combinatorial problem in the symmetric group ............................... 175

Rodolfo DeSapio, Unknotting spheres via Smale .................. 179

James E. Shockley, On the functional equation

$$
F(m n) F((m, n))=F(m) F(n) f((m, n)) \ldots \ldots \ldots
$$

Kenneth Edward Whipple, Cauchy sequences in Moore spaces ... 